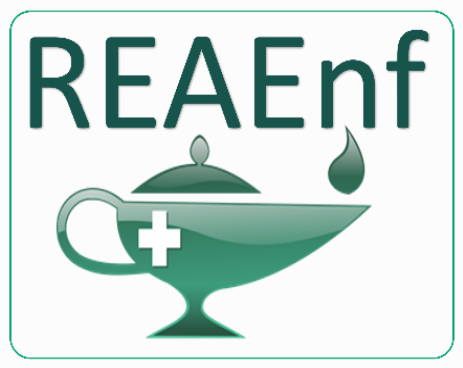

Revista Eletrônica Acervo Enfermagem
REVISÃo BIBLIOGRÁFICA

Recebido em: 10/2020

Aceito em: 11/2020

Publicado em: 12/2020

\title{
Impactos da política nacional de atenção básica nas taxas de mortalidade infantil sob a ótica da estratégia de saúde da família
}

\author{
Impacts of the national basic care policy on child mortality rates from the family health \\ strategy
}

Impactos de la política nacional de atención básica en las tasas de mortalidad infantil de la estrategia de salud de la familia

Glayse Xavier Gonçalves dos Santos ${ }^{1 *}$, Simone Pereira da Silva ${ }^{1}$, José William Araújo do Nascimento ${ }^{2 *}$, Diógenes José Gusmão Coutinho${ }^{1}$.

\begin{abstract}
Resumo: Este estudo buscou analisar os impactos da Política Nacional de Atenção Básica (PNAB) nas taxas de mortalidade infantil no âmbito da Estratégia de Saúde da Família (ESF). Para isso foi realizado uma revisão integrativa na Biblioteca Virtual em Saúde por meio do acesso simultâneo às bases de dados BDENF, CVSP e LILACS, utilizando os seguintes descritores: "Estratégia Saúde da Família" e "Mortalidade Infantil". 49 publicações foram encontradas inicialmente, porém apenas oito artigos foram incluídos na análise final. Verificou-se uma redução drástica da taxa de mortalidade infantil a partir do momento de expansão da ESF pelas regiões mais carentes do Brasil, favorecendo uma queda deste indicador com consequente melhoria na qualidade da assistência à saúde e na qualidade de vida, pondo o Brasil com uma das nações de maior evolução neste âmbito, proporcionada principalmente pelas ações que a PNAB desenvolveu ao longo dos anos. Desta forma, as publicações analisadas por esta revisão demonstraram que a PNAB contribui para que as taxas de mortalidade infantil alcancem valores ainda menores, principalmente pelas ações desempenhadas pelas equipes de saúde em especial os enfermeiros.
\end{abstract}

Palavras-chave: Atenção primária à saúde, Mortalidade infantil, Política pública.

\begin{abstract}
This study sought to analyze the impacts of the National Primary Care Policy (PNAB) on infant mortality rates within the scope of the Family Health Strategy (ESF). For this purpose, an integrative review was carried out at the Virtual Health Library through simultaneous access to the BDENF, CVSP and LILACS databases, using the following descriptors: "Family Health Strategy" and "Child Mortality". 49 publications were found initially, but only eight articles were included in the final analysis. There was a drastic reduction in the infant mortality rate from the moment of expansion of the FHS by the poorest regions of Brazil, favoring a drop in this indicator with a consequent improvement in the quality of health care and quality of life, putting Brazil with one of the most evolving nations in this area, provided mainly by the actions that PNAB has developed over the years. In this way, the publications analyzed by this review demonstrated that the PNAB contributes to infant mortality rates reaching even lower values, mainly due to the actions performed by the health teams, especially nurses.
\end{abstract}

Keywords: Primary health care, Infant mortality, Public policy.

${ }^{1}$ Faculdade Alpha, Recife - PE.

2Universidade Católica de Pernambuco, Recife - PE. *E-mail: jwanb4321@gmail.com 
Resumen: Este estudio buscó analizar los impactos de la Política Nacional de Atención Primaria (PNAB) sobre las tasas de mortalidad infantil en el ámbito de la Estrategia de Salud de la Familia (ESF). Se realizó una revisión integradora en la Biblioteca Virtual en Salud mediante el acceso a las bases de datos BDENF, CVSP y LILACS, utilizando los siguientes descriptores: "Estrategia Salud de la Familia" y "Mortalidad Infantil". Inicialmente se encontraron 49 publicaciones, pero solo se incluyeron ocho artículos en el análisis final. Hubo una drástica reducción en la tasa de mortalidad infantil desde el momento de la expansión de la ESF por las regiones más pobres de Brasil, favoreciendo una caída en este indicador con una consecuente mejora en la calidad de la atención de salud y la calidad de vida, colocando a Brasil con una de las naciones más evolucionadas en esta área, proporcionada por las acciones que la PNAB ha desarrollado a lo largo de los años. De esta manera, las publicaciones analizadas por esta revisión demostraron que la PNAB contribuye a que las tasas de mortalidad infantil alcancen valores aún más bajos, principalmente por las acciones realizadas por los equipos de salud, especialmente enfermeras.

Palabras clave: Atención primaria de salud, Mortalidad infantil, Políticas públicas.

\section{INTRODUÇÃO}

O modelo de saúde encontrado atualmente no Brasil passou por várias mudanças, que teve marco inicial histórico pela criação do Sistema Único de Saúde (SUS), instituído pela Constituição Federal (CF) de 1988 e consolidado pelas Leis 8.080 e 8.142 . Este sistema surgiu como uma nova forma de política sócio organizacional, com características peculiares, a exemplo do mais essencial, que diz respeito à colocação constitucional de que Saúde é Direito do Cidadão e Dever do Estado (CARVALHO G, 2013).

Nesse contexto a assistência à saúde passa a ser vista como uma política pública redistributiva e para que essas mudanças preconizadas na elaboração e implementação do SUS ocorressem na prática nos serviços de saúde, o Ministério da Saúde (MS) passou a reforçar suas estratégias de ação com foco na Atenção Básica à Saúde (ABS), também denominada de Atenção Primária à Saúde (APS) (FREY K, 2000; SOUZA MLBS; MELO CAV, 2013).

O Programa de Saúde da Família (PSF), criado em 1994, gradualmente passou a se tornar uma estratégia eficaz que pudesse ampliar o acesso da população à ABS bem como em transformar o modelo assistencial vigente, e para isso ao longo dos anos ela foi se transformando mediante a necessidade de organizar o sistema de saúde brasileiro conforme medidas solicitadas pela Organização Mundial da Saúde (OMS) (OMS, 2008).

Em 2006 então, foi publicada a primeira versão da Política Nacional de Atenção Básica (PNAB), com a finalidade de estabelecer as diretrizes organizacionais, dando início a uma importante discussão na área de políticas públicas (BRASIL, 2006a). A partir de sua criação, o PSF passou a ser um eixo norteador e estruturante do SUS, sendo transformado em Estratégia de Saúde da Família (ESF), que se consolidou e expandiu-se com o objetivo de reorganizar a Atenção Básica (AB) brasileira (BRASIL, 2006b).

Ela trouxe um norte com várias recomendações para os serviços de saúde, envolvendo processos de trabalho, composição das equipes de saúde, bem como suas atribuições, financiamento do sistema, dentre outras recomendações de suma importância para ABS (BRASIL, 2006b). A PNAB ampliou a concepção definida de $A B$, passando a reconhecer a Saúde da Família (SF) como uma forma que pudesse substituir o modelo desta Atenção até então praticado, revisando as funções das Unidades Básicas de Saúde (UBS). Devido a necessidade da adequação desta política, duas revisões foram realizadas, em 2011 e 2017, ou seja, a PNAB conta com três versões, que foram produzidas em situações sociais e financeiras distintas do Brasil (GOMES CBS, et al., 2020).

Ela difundiu no país alguns conceitos estabelecidos por Starfield B (2002), como primeiro contato, longitudinalidade, integralidade, coordenação, orientação para a comunidade, centralidade na família e competência cultural, e sua implantação causou diversos benefícios à saúde da família brasileira, demonstrado por diversos estudos. 
Um dos principais impactos da PNAB na ESF foi a sua relação com a taxa de mortalidade infantil, indicador este que representa um importante marcador de análise da saúde de uma determinada população, podendo também ser utilizada para aferir os resultados alcançados pela ESF (CECCON RF, et al., 2014).

A taxa de mortalidade infantil se trata de um indicador que expressa a combinação de vários fatores individuais ou coletivos que se relacionam à saúde de crianças menores de 1 ano, sendo este um indicador que expressa com veemência a qualidade de vida de um país. Nos últimos anos tem se observado uma redução das taxas de mortalidade infantil, seja por esforços nacionais ou internacionais para uma melhor assistência à gestante e ao recém-nascido, especialmente no âmbito da Atenção Primária à Saúde (LOURENÇO EC, et al., 2014).

Estudo conduzido entre o Ministério da Saúde e a Universidade de Nova York verificou que o modelo de atenção à saúde brasileiro, principalmente seu marco estratégico, a Estratégia de Saúde da Família, foi decisivo para que houvesse uma diminuição nas taxas de mortalidade infantil no Brasil, mais até do que o acesso aos hospitais e maior oferta de médicos, e isto teve um ápice a partir da implementação da PNAB, que ampliou e aumentou a qualidade da cobertura de saúde à população brasileira (BRASIL, 2008).

Isto porque a ESF se fundamenta em conceitos que norteiam a saúde do país como é o caso da integralidade da assistência, de modo que o atendimento prestado não se restringe apenas ao indivíduo ou ao que ele apresenta, mas também a toda a sua coletividade e a partir da análise de outros aspectos como determinantes ambientais, culturais, sociais e econômicos que sejam específicos da comunidade de abrangência da ESF. Esta visão ampliada e integralizada tem impacto direto nas taxas de mortalidade infantil dado o acompanhamento contínuo que abrange os sujeitos envolvidos (LOURENÇO EC, et al., 2014).

A ESF tem contribuído significativamente para a redução da mortalidade infantil e da evasão escolar no Brasil, e isto pode ser evidenciado pela conclusão do estudo Impactos da Estratégia Saúde da Família e Desafios para o Desenvolvimento Infantil (2019), onde verificou-se que a queda da mortalidade infantil é proporcional à cobertura da ESF e é mais evidente nas regiões Norte e Nordeste do país, sendo mais evidente especialmente após a implantação da PNAB (NCPI, 2019). Isto se deu principalmente devido as ações das equipes de enfermagem na ESF, onde o cuidado prestado pelos enfermeiros abrange não apenas no foco individual ou curativo, mas principalmente ações de prevenção e promoção à saúde, desenvolvidas tanto nas consultas como em lares, escolas, igrejas e outros espaços comunitários, envolvendo os sujeitos, família e comunidade, desde a menor a mais alta faixa etária, nas diferentes situações do processo saúde-doença (NETO FRGX, et al., 2011).

Desta forma, a fim de demonstrar os impactos que esta política causou nas taxas de mortalidade infantil por meio da Estratégia de Saúde da Família desde a sua implantação, este estudo objetivou analisar os principais impactos da Política Nacional de Atenção Básica na taxa de mortalidade infantil sob a ótica da Estratégia de Saúde da Família.

\section{MÉTODOS}

Foi realizado um estudo de revisão integrativa da literatura baseada em uma análise crítica. A pesquisa foi realizada a partir das seguintes etapas, idealiza por Souza MT, et al. (2010): 1) Definição da questão norteadora e objetivos da pesquisa; 2) Estabelecimento dos critérios de inclusão e exclusão/ amostragem dos estudos; 3) Busca na literatura; 4) Categorização e análise dos estudos; 5) Apresentação e discussão dos resultados da amostra e; 6) Apresentação e síntese do conhecimento.

A questão norteadora deste estudo foi: "Quais os impactos da Política Nacional de Atenção Básica nas taxas de mortalidade infantil sob a ótica da Estratégia de Saúde da Família?' A investigação dos estudos ocorreu na Biblioteca Virtual em Saúde por meio do acesso simultâneo às seguintes bases de dados: Base de Dados de Enfermagem (BDENF), Campus Virtual de Saúde Pública (CVSP) e Literatura Latinoamericana e do Caribe em Ciências da Saúde (LILACS), no período de setembro a outubro de 2020. 
Também foram usados como base para apresentação dos resultados outras referências de importância para abordagem do tema, como materiais do Ministério da Saúde e livros.

Para consulta dos artigos foram usadas palavras-chaves extraídas dos Descritores em Ciências da Saúde (DeCS). Foram utilizados os seguintes descritores: "Estratégia Saúde da Família" e "Mortalidade Infantil", por meio do operador booleano "AND". Com relação aos artigos selecionados, estes atenderam aos seguintes critérios de inclusão: pesquisas disponíveis eletronicamente nas bases de dados selecionadas, publicadas em português e disponíveis na íntegra. Foi estabelecido um recorte temporal entre 2006 e 2020, uma vez que a PNAB teve sua primeira versão publicada em 2006. Foram excluídos os artigos duplicados nas bases de dados (duplicatas), estudos com temática não relevante a questão norteadora da pesquisa e artigos ou documentos publicados antes de 2006.

Para garantir o registro do conjunto de informações relevantes ao tema, foi utilizado instrumento proposto por Souza MAO, et al. (2018), adaptado para este estudo com as seguintes variáveis: dados de identificação geral (título, autores, periódico, ano de publicação), delineamento metodológico e principais resultados específicos. Após a aplicação dos filtros de pesquisa na BVS, inicialmente foram encontrados 49 artigos, de modo que os artigos duplicados foram registrados apenas uma vez, totalizando em 44. Após a leitura dos títulos e resumos simultaneamente foram excluídas 26 publicações. Sendo assim, 18 publicações foram selecionadas para leitura na íntegra, porém 10 foram excluídas. Assim, a amostra final foi constituída por oito artigos, conforme se observa na Figura 1.

Figura 1 - Fluxograma de seleção dos estudos.

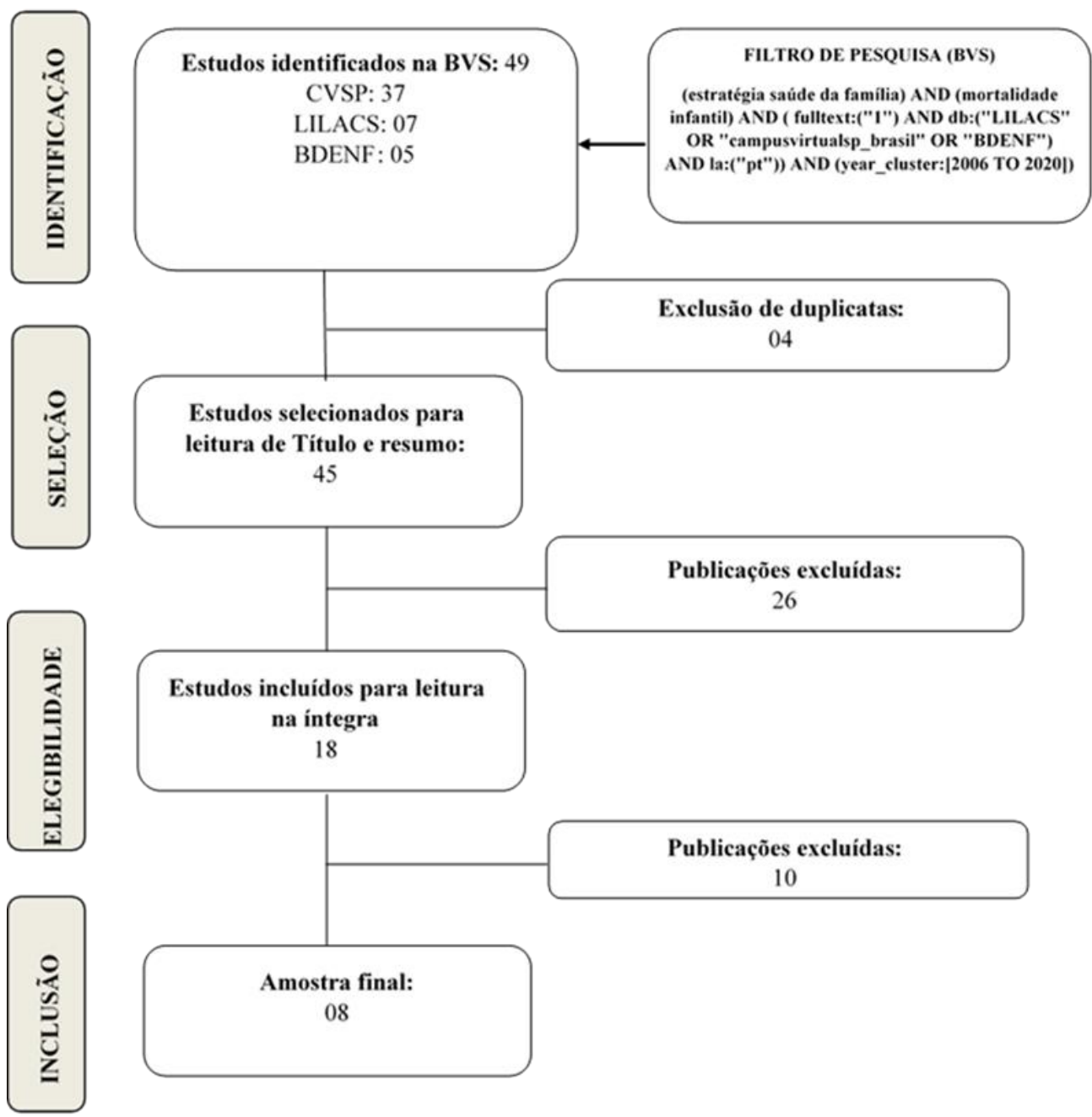

Fonte: Santos GXG, et al., 2020. 
Este estudo preservou os aspectos éticos de tal forma que todos os autores das publicações analisadas foram referenciados apropriadamente, mediante a Lei de Direitos Autorais no 9.610 de 19 de Fevereiro de 1998 (BRASIL, 1998).

\section{RESULTADOS}

Do total de oito artigos analisados, seis estavam indexados na LILACS e dois na CVSP. Observa-se, no Quadro 1, maior frequência de publicação nos anos de 2014, 2016 e 2018, com duas publicações cada. Referente à abordagem dos artigos encontrados, verifica-se que a maior parte utilizou o estudo ecológico de abordagem retrospectiva ( $n$ : 4). Todos os estudos objetivaram de forma direta ou indireta avaliar o impacto da ESF na taxa de mortalidade infantil após a implementação da PNAB.

Quadro 1 - Caracterização geral dos artigos da amostra.

\begin{tabular}{|c|c|c|c|}
\hline $\begin{array}{l}\text { Primeiro } \\
\text { autor, ano }\end{array}$ & Periódico & Objetivos & Tipo de estudo \\
\hline $\begin{array}{l}\text { Sousa MLB, } \\
2013\end{array}$ & $\begin{array}{l}\text { Revista Política } \\
\text { Hoje }\end{array}$ & $\begin{array}{l}\text { Avaliar o impacto da PNAB na taxa de } \\
\text { mortalidade infantil nos municípios brasileiros. }\end{array}$ & Retrospectivo \\
\hline $\begin{array}{l}\text { Lourenço EC, } \\
2014\end{array}$ & $\begin{array}{l}\text { Rev Saude } \\
\text { Publica }\end{array}$ & $\begin{array}{l}\text { Verificar a relação entre o modelo de atenção e } \\
\text { ao coeficiente de mortalidade infantil, no estado } \\
\text { de São Paulo, no período de } 1998 \text { a } 2008 .\end{array}$ & $\begin{array}{l}\text { Ecológico } \\
\text { retrospectivo }\end{array}$ \\
\hline $\begin{array}{l}\text { Ceccon RF, } \\
\quad 2014\end{array}$ & $\begin{array}{l}\text { Cad. Saúde } \\
\text { Colet }\end{array}$ & $\begin{array}{c}\text { Analisar a relação entre mortalidade infantil e } \\
\text { cobertura populacional da Saúde da Família nas } \\
\text { unidades da Federação brasileira. }\end{array}$ & $\begin{array}{l}\text { Ecológico } \\
\text { retrospectivo }\end{array}$ \\
\hline $\begin{array}{l}\text { Gomes } \\
\text { TGACB, } 2016\end{array}$ & $\begin{array}{l}\text { Comun. ciênc. } \\
\text { saúde }\end{array}$ & $\begin{array}{c}\text { Analisar as tendências da mortalidade infantil no } \\
\text { Brasil e regiões com a cobertura da ESF }\end{array}$ & Epidemiológico \\
\hline $\begin{array}{l}\text { Venancio SI, } \\
\quad 2016\end{array}$ & $\begin{array}{l}\text { Rev. Bras. } \\
\text { Saude Mater. } \\
\text { Infant }\end{array}$ & $\begin{array}{l}\text { Avaliar a efetividade da ESF sobre indicadores } \\
\text { de saúde da criança no Estado de São Paulo. }\end{array}$ & $\begin{array}{l}\text { Ecológico } \\
\text { retrospectivo }\end{array}$ \\
\hline $\begin{array}{l}\text { Carneiro VB, } \\
2018\end{array}$ & $\begin{array}{l}\text { Ciênc. saúde } \\
\text { coletiva }\end{array}$ & $\begin{array}{c}\text { Avaliar o desempenho da ESF após a } \\
\text { implantação do Programa Mais Médicos no } \\
\text { território do Marajó-PA. }\end{array}$ & $\begin{array}{l}\text { Ecológico } \\
\text { retrospectivo }\end{array}$ \\
\hline $\begin{array}{l}\text { Silva ESA, } \\
\quad 2018\end{array}$ & Saúde Debate & $\begin{array}{c}\text { Avaliar o impacto da ESF sobre a mortalidade } \\
\text { infantil do Semiárido brasileiro. }\end{array}$ & $\begin{array}{l}\text { Ecológico } \\
\text { longitudinal }\end{array}$ \\
\hline $\begin{array}{l}\text { Silva VC, } \\
2019\end{array}$ & Rev. Econ. NE & $\begin{array}{l}\text { Avaliar o impacto do Programa de Saúde da } \\
\text { Família no Nordeste do Brasil. }\end{array}$ & Retrospectivo \\
\hline
\end{tabular}

Fonte: SANTOS GXG, et al., 2020.

As relações estabelecidas entre a ESF e a taxa de mortalidade infantil após a implementação da PNAB são evidenciadas pelo Quadro 2, onde verifica-se uma redução drástica desta taxa a partir do momento de expansão da ESF pelas regiões mais carentes do Brasil, favorecendo uma queda deste indicador com consequente melhoria na qualidade da assistência à saúde e na qualidade de vida, pondo o País com uma das nações de maior evolução neste âmbito, proporcionada principalmente pelas ações que a PNAB desenvolveu ao longo dos anos.

Os estudos analisados apresentam análises estatísticas relevantes que apontam que a ESF diminuiu a taxa de mortalidade infantil ao longo destes anos, como resultado especial das políticas públicas implementadas no território brasileiro. 
Quadro 2 - Relações entre a expansão da ESF após a PNAB com a taxa de mortalidade infantil.

\begin{tabular}{|c|c|}
\hline Primeiro autor, ano & Principais resultados \\
\hline Sousa MLB, 2013 & $\begin{array}{c}\text { Verificou-se que os municípios brasileiros que não estavam abrangidos pela ESF } \\
\text { tiveram taxa de mortalidade infantil superior a } 10 \% \text { em comparação aqueles que } \\
\text { tinham, apontando para uma relação de causalidade negativa entre ESF e taxa } \\
\text { de mortalidade infantil. }\end{array}$ \\
\hline Lourenço EC, 2014 & $\begin{array}{l}\text { Houve diminuição da mortalidade infantil no decorrer dos anos }(p<0,05) \text {, de } \\
\text { modo que a proporção de ESF implantado }(p<0,0001) \text { foram significativos no } \\
\text { modelo. A queda da mortalidade infantil no período analisado foi influenciada pelo } \\
\text { crescimento do PIB per capita e pelo modelo Saúde da Família. }\end{array}$ \\
\hline Ceccon RF, 2014 & $\begin{array}{c}\text { A expansão da cobertura da ESF esteve associada à redução das taxas de } \\
\text { mortalidade infantil em } 73 \% \text { dos Estados brasileiros }(p<0,05) .\end{array}$ \\
\hline $\begin{array}{l}\text { Gomes TGACB, } \\
2016\end{array}$ & $\begin{array}{c}\text { A cobertura populacional realizada pela ESF foi maior nas regiões com as } \\
\text { maiores taxas de mortalidade infantil (Norte e Nordeste) as quais demonstraram } \\
\text { maiores decréscimos. Os dados apontam que a expansão da ESF, juntamente } \\
\text { com outras melhorias em condições socioeconômicas, está associada a reduções } \\
\text { na mortalidade na infância. }\end{array}$ \\
\hline Venancio SI, 2016 & $\begin{array}{l}\text { Em 1998, a taxa de mortalidade infantil no Estado de São Paulo era de } 18,67 \text { e } \\
\text { alcançou, em } 2009 \text { (após a PNAB) } 12,48 \text { por mil nascidos vivos. } \\
\text { Concomitantemente verificou-se um efeito protetor da ESF em relação a diarreia } \\
\text { e a pneumonia. }\end{array}$ \\
\hline Carneiro VB, 2018 & $\begin{array}{l}\text { A taxa de mortalidade infantil demonstrou uma tendência decrescente a partir do } \\
\text { segundo semestre de } 2014 \text {, na maioria dos municípios e região do Marajó. }\end{array}$ \\
\hline Silva ESA, 2018 & $\begin{array}{l}\text { As ações envolvendo políticas públicas especialmente a ampliação da ESF } \\
\text { reduziu os níveis de fecundidade e mortalidade infantil no semiárido brasileiro. }\end{array}$ \\
\hline Silva VC, 2019 & $\begin{array}{l}\text { O principal resultado obtido foi que o impacto da política sobre a redução da } \\
\text { mortalidade infantil é significante, ou seja, quanto maior o percentual de cobertura } \\
\text { da ESF, menor o número de óbitos infantis. }\end{array}$ \\
\hline
\end{tabular}

Fonte: Santos GXG, et al., 2020.

\section{DISCUSSÃO}

As publicações analisadas por esta pesquisa se fundamentam como pesquisas bases acerca dos impactos diretos da PNAB nas taxas de mortalidade infantil no âmbito da ESF em diversos municípios do Brasil. Esta temática é de suma importância uma vez que esta taxa se refere ao mais tradicional indicador de saúde, representando um risco que um nascido vivo tem em ir a óbito em seu primeiro ano de vida. Os indicadores de queda da taxa de mortalidade infantil têm diversos determinantes neste processo, incluindo as ações sociais e políticas. Dentre estas ações está a implantação da PNAB que reformulou o PSF, o estruturando como uma estratégia, marco que fundamentou a Atenção Primária à Saúde. As evidências geradas sugerem que o impacto desta política é significativo e positivo sobre o aumento da expectativa de vida ao nascer (MACINKO J, et al., 2007). O decréscimo destes indicadores ocorreu no mesmo período da expansão da APS por meio da ESF, onde houve um aumento da população brasileira assistida por este modelo de atenção, permeando um aumento no acompanhamento das gestantes pelos profissionais da saúde, instruindo estas mulheres e suas famílias acerca da prevenção de doenças (SILVA VC, et al., 2019). 
Em 1995 o PSF estava presente e apenas 115 municípios e quando se observa em 2011, mais de 94\% dos municípios brasileiros tinham cobertura da então ESF. Esta estratégia contribui de maneira significativa para redução da mortalidade infantil, de modo que os municípios com mais de $70 \%$ de cobertura da ESF tinham $34 \%$ menos crianças com baixo peso ao nascer, além de melhor cobertura vacinal. A implementação do ESF estava associada a reduções na mortalidade, principalmente nas fases iniciais da vida, devido à intervenção nas condições perinatais, doenças infecciosas, endócrinas e respiratórias (GOMES TGACB, et al., 2016).

Assim que a PNAB foi implantada em 2006, já em 2007 a taxa de mortalidade infantil decaiu de 19,6\% em 2005 para 18,3\%, ao passo que a cobertura da ESF passou de 58,7\% em 2005 para $67,8 \%$ em 2007, sendo consequência direta da PNAB pelo fortalecimento da APS (SILVA VC, et al., 2019). Dados do estudo de Sousa MLBS e Melo CAV (2013) revelaram que os municípios que tinham a ESF implantada no município tiveram uma taxa de mortalidade infantil inferior em aproximadamente $10 \%$ quando comparados aos municípios desprovidos. O incentivo da PNAB também teve como foco as campanhas de vacinação como prioridades na ESF ou na APS como um todo, causando impactos positivos na redução da taxa mortalidade infantil (FRANÇA ISX, et al., 2009).

No Brasil, a taxa de mortalidade infantil de 2017 foi menor que 14,4 mortes por 1.000 nascidos vivos, sendo que $64,7 \%$ foram por causas evitáveis. Essa taxa de mortalidade foi atingida após uma trajetória de forte redução nesse indicador, uma vez que antes da PNAB os valores eram em torno de 43 mortos por 1.000 nascidos vivos (BRASIL, 2017).

Por mais que a ESF tenha proporcionado esta redução tão acentuada, ainda assim, o País precisa percorrer um longo caminho para alcançar os níveis das regiões mais desenvolvidas do mundo, que é de, aproximadamente, cinco óbitos para cada mil nascidos vivos. Entre as regiões brasileiras, o Nordeste avançou expressivamente com relação à diminuição do nível da taxa de mortalidade infantil, isto porque esta região por muito tempo foi desprovida de uma assistência elevada, algo que passou a ocorrer por meio da expansão das equipes de saúde da família a regiões mais carentes, especialmente nos municípios mais carentes e zona rural (SILVA ESA, et al., 2018).

Uma das ações da PNAB foi a implantação do Programa Mais Médicos, fixando médicos em regiões isoladas com carência de profissionais, por meio do seu componente emergencial de provimento. Conforme o estudo realizado por Carneiro VB, et al. (2018) na região do Marajó, região esta que já apresentou taxa de mortalidade infantil superior à do Estado do Pará, a implantação do programa causou uma redução dos índices desta taxa. Porém as atividades exercidas pelos enfermeiros da ESF desempenham papel fundamental para que todas as taxas de mortalidade infantil pudessem ser reduzidas em todas as regiões do país. As ações realizadas por estes profissionais promovem estímulo ao aleitamento materno, alimentação saudável, imunização, prevenção de doenças prevalentes na infância, além de várias outras ações que o tornam sujeitos principais na qualidade de vida da população (SILVA GN e CARDOSO AM, 2018).

\section{CONSIDERAÇÕES FINAIS}

A partir desta revisão da literatura verificou-se que a Política Nacional de Atenção Básica promoveu uma reestruturação da Estratégia de Saúde da Família, fortalecendo a Atenção Primária à Saúde e expandindo este novo modelo de assistência para todas as regiões do país, com uma cobertura que se aproxima dos 100\%.A partir deste momento várias ações no âmbito da ESF passaram a ser realizadas com o intuito de promover mudanças na saúde brasileira e uma delas se refere a uma redução drástica nas taxas de mortalidade infantil. A expansão desta estratégia proporcionou que o país evoluísse bastante de modo que a taxa de mortalidade infantil é o indicador mais forte na qualidade de assistência à saúde. Desta forma, as publicações analisadas por esta revisão demonstraram que a PNAB em suas três versões, contribuiu e ainda contribui para que as taxas de mortalidade infantil alcancem valores ainda menores, principalmente pelas ações desempenhadas pelas equipes de saúde em especial os enfermeiros, que exercem papel fundamental na promoção e prevenção da saúde brasileira. 


\section{REFERÊNCIAS}

1. BRASIL. Ministério da Saúde (MS). Lei no 9.610, de 19 de fevereiro de 1998, que altera, atualiza e consolida a legislação sobre direitos autorais e dá outras providências. Diário Oficial da União. Brasília: Ministério da Saúde; 1998.

2. BRASIL. Ministério da Saúde (MS). Gabinete do Ministro. Portaria no 399/GM, de 22 de fevereiro de 2006. Divulga o Pacto pela Saúde 2006-Consolidação do SUS e aprova as Diretrizes Operacionais do referido Pacto. Diário Oficial da União 2006a.

3. BRASIL. Ministério da Saúde (MS). Portaria/MS no 648, de 28 de março de 2006. Aprova a Política Nacional de Atenção Básica, estabelecendo a revisão de diretrizes e normas para a organização da Atenção Básica para o Programa Saúde da Família (PSF) e o Programa Agentes Comunitários de Saúde (PACS). Diário Oficial da União 2006b.

4. BRASIL. Ministério da Saúde (MS). Sistema Único de Saúde. Painel de Indicadores do SUS n 4. Temático Saúde da Família v II. Brasília: MS; 2008b

5. BRASIL. Ministério da Saúde (MS). Dados do Sistema de Informação sobre Mortalidade e do Sistema de Informação sobre Nascidos Vivos, do Datasus/Ministério da Saúde; 2017.

6. CARNEIRO VB, et al. Tecobé no Marajó: tendência de indicadores de monitoramento da atenção primária antes e durante o Programa Mais Médicos para o Brasil. Ciênc. saúde coletiva, 2018; 23(7): 2413-2422.

7. CARVALHO G. A saúde pública no Brasil. Estudos avançados, 2013; 27(78): 1-22.

8. CECCON RF, et al. Mortalidade infantil e Saúde da Família nas unidades da Federação brasileira, 1998-2008. Cad. Saúde Colet, 2014; 22(2): 177-183.

9. FRANÇA ISX, et al. Cobertura vacinal e mortalidade infantil em Campina Grande, PB, Brasil. Revista Brasileira de Enfermagem, 2009; 62(21): 258-264.

10. FREY K. Políticas públicas: um debate conceitual e reflexões referentes à prática da análise de políticas públicas no Brasil. Planejamento e Políticas públicas, 2000; 1(21): 211-259.

11. GOMES TGACB, et al. Mortalidade na infância no Brasil e regiões no período de 2000 a 2011: o impacto da atenção básica. Com. Ciências Saúde, 2016; 27(4): 259-266.

12. GOMES CBS, et al. Política Nacional de Atenção Básica de 2017: análise da composição das equipes e cobertura nacional da Saúde da Família. Ciênc. saúde coletiva, 2020; 25(4).

13. ORGANIZAÇÃO MUNDIAL DA SAÚDE (OMS). Relatório Mundial de Saúde 2008. Cuidados de Saúde Primários: agora mais do que nunca. Editor da versão Portuguesa: Alto Comissariado da Saúde, Ministério da Saúde de Portugal. OMS; 2008.

14. LOURENÇO EC, et al. Variáveis de impacto na queda da mortalidade infantil no Estado de São Paulo, Brasil, no período de 1998 a 2008. Rev Saude Publica, 2014; 19(7): 2055-2062.

15. MACINKO J, et al. Evaluation of the impact of the Family Health Program on infant mortality in Brazil, 1990-2002. Journal of Epidemiology and Community Health, 2007; 60: 13-19.

16. NETO FRGX, et al. Práticas do enfermeiro da Estratégia Saúde da Família na atenção à saúde da criança, Cariré Ceará. Rev. Soc. Bras. Enferm. Ped, 2011;11(1): 9-16.

17. NÚCLEO CIÊNCIA PELA INFÂNCIA (NCPI). Estudo impactos da estratégia saúde da família e desafios para o desenvolvimento infantil: estudo 5 / Comitê Científico Núcleo Ciência pela Infância. — São Paulo: NCPI, 2019.

18. PEREIRA MG. Artigos científicos: como redigir, publicar e avaliar. Rio de Janeiro: Guanabara Koogan; 2011.

19. SILVA GN, CARDOSO AM. O papel do enfermeiro na redução da mortalidade infantil por meio do acompanhamento de puericultura na Atenção Básica. Rev Cien Escol Estad Saud Publ Cândido Santiago-RESAP, 2018; 4(1): 91-99.

20. SILVA ESA, et al. Efeitos dos programas governamentais e da fecundidade sobre a mortalidade infantil do Semiárido brasileiro. Saúde debate, 2018; 42(116): 138-147.

21. SILVA VC, et al. Avaliação do impacto do Programa Saúde da Família sobre a mortalidade infantil no Nordeste do Brasil. Rev. Econ. NE, 2019; 50(3): 39-48.

22. SOUSA MLBS, MELO CAVM. Impacto da Política de Atenção Básica à Saúde na Taxa de Mortalidade Infantil nos Municípios Brasileiros. Revista Política Hoje, 2013; 22(1): 250-276.

23. SOUZA MT, et al. Integrative review: what is it? How to do it? Einstein (São Paulo), 2010; 8(1): 102-106.

24. SOUZA MAO, et al. Odor evaluation scales for odor in neoplastic wounds: an integrative review. Rev Bras Enferm, 2018; 71(5): 2552-2560.

25. STARFIELD B. Atenção primária: equilíbrio entre necessidades de saúde, serviços e tecnologia. Brasília: UNESCO, Brasil. Ministério da Saúde ed.; 2002.

26. VENANCIO SI, et al. Efetividade da Estratégia Saúde da Família sobre indicadores de saúde da criança no Estado de São Paulo. Rev. Bras. Saude Mater. Infant, 2016;16(3). 